\title{
DualEELS at High Energy Losses: Exploring Lower Accelerating Voltages and Absolute Cross Sections
}

\author{
Ian MacLaren ${ }^{1}$, Hidetaka Sawada ${ }^{2}$, Samuel McFadzean ${ }^{1}$, Luke Gardiner ${ }^{1}$, Katherine E. MacArthur ${ }^{3}$ and \\ Alan J. Craven ${ }^{1}$ \\ 1. School of Physics and Astronomy, University of Glasgow, Glasgow, Scotland, UK. \\ 2. JEOL House, Silver Court, Watchmead, Welwyn Garden City, Herts., UK. \\ 3. Ernst Ruska-Centre for Microscopy and Spectroscopy with Electrons and Peter Grünberg Institute \\ Forschungszentrum Jülich, Germany.
}

EELS has typically been performed at relatively low energy losses. The majority of studies are at losses of less than $1 \mathrm{kV}$ and there are quite a few between 1 and $2 \mathrm{kV}$. High quality studies above $2 \mathrm{kV}$ are rarer, and above $3 \mathrm{kV}$ there are fewer than ten published studies known to the authors. Apart from the obvious point that there is relatively little signal out there, and therefore counting times will be long, there are also electron optical issues that make transferring the signal into the spectrometer more difficult with increasing loss. Specifically, the electron optics of STEM columns are often not explicitly tuned to transfer high energy losses into the spectrometer with the same efficiency as the zero loss electrons. Recently, we reported the detailed characterisation and optimisation of the post-specimen lenses in a modern aberration-corrected STEM for spectroscopy, nominally out to $3 \mathrm{kV}$ at $200 \mathrm{kV}$ beam energy [1]. We then reported some possible applications of this high loss EELS [2]. In this paper, we report further advances in extending high loss EELS to $80 \mathrm{kV}$ beam energy, and in using high loss Dual EELS at $200 \mathrm{kV}$ to measure absolute cross sections for some Pt-group metals.

Following our earlier work at $200 \mathrm{kV}$, we have optimized our $2 \mathrm{~cm}$ camera length at $80 \mathrm{kV}$ to improve the transfer of loss electrons into the spectrometer. Figure 1a shows a spectrum recorded with the original $2 \mathrm{~cm}$ camera length. As seen previously at $200 \mathrm{kV}$ [1], extra background intensity perturbs the background shape, here from about $1500 \mathrm{eV}$, making EELS analysis of $\mathrm{Si}$ or other semiconductors problematic. Figure $1 \mathrm{~b}$ shows that the problem is fixed with the new $2 \mathrm{cmC}$ camera length. Figure 1c) shows why. For the $2 \mathrm{~cm}$ camera length, the EELS stripe folds inwards rather rapidly, and extra intensity from the edges is folded into the EELS entrance aperture. A crossover occurs around $3500 \mathrm{eV}$. For the optimized $2 \mathrm{cmC}$ camera length, this effect is much delayed with the crossover happening between 5000 and $5500 \mathrm{eV}$, potentially allowing good quality EELS out to close to $3000 \mathrm{eV}$.

Previously, we reported characterization of the $\mathrm{L}_{2,3}$ EELS edges of some $2^{\text {nd }}$ row transition elements, including changes with oxidation and calculation of absolute differential cross sections of two $2^{\text {nd }}$ row transition elements: $\mathrm{Zr}$ and $\mathrm{Mo}[2,3]$. We now extend this to three further $2^{\text {nd }}$ row transition elements, all of which are platinum group metals with applications in catalysis: $\mathrm{Ru}, \mathrm{Rh}$ and $\mathrm{Pd}$. The cross sections were calculated as in our previous publications [3,4] using DualEELS to provide a low loss spectrum to go with the core-loss spectrum. This allows deconvolution of plural scattering, normalization by the zero loss intensity, and determination of thickness to allow normalization by the areal density of atoms. To allow for optimal sharpness of both the zero loss peak and the core loss edges, two datasets were recorded for each area, one using an $\mathrm{F}_{\mathrm{x}}$ value for the spectrometer suitable for the low loss, and the other used one optimized for the core loss, as described previously [3]. The $\mathrm{F}_{\mathrm{x}}$ change for the core loss was +0.4 in the GIF Quantum for all datasets shown here. Figure 2 shows background-subtracted cross sections for the three elements. Both $\mathrm{Ru}$ and $\mathrm{Rh}$ show significant white lines at the $\mathrm{L}_{3}$ and $\mathrm{L}_{2}$ edges, as 
is typical for transition elements due to the significant density of empty $d$-states just above the Fermi level. Rh, however, shows a significant decline in the white line intensity relative to the continuum background as compared to $\mathrm{Ru}$ - this is entirely expected as there will be fewer empty $d$-states per atom in $\mathrm{Rh}$ - there are nominally 2 empty $d$-states per atom in Rh as opposed to 3 per atom in $\mathrm{Ru}$. Pd, on the other hand, shows no strong white lines, which accords well with simple chemistry models of the isolated atom electronic structure of $[\mathrm{Kr}] 4 d^{10}$ leaving no empty $d$-states whatsoever. This trend of decreasing and finally disappearing white lines is rather similar to that previously observed in $\mathrm{L}_{2,3}$ edges for $1^{\text {st }}$ row transition elements from Sc-Zn $[5,6]$.

\section{References:}

[1] AJ Craven et al, Ultramicroscopy 180 (2017), p. 66.

[2] KJ Annand, I MacLaren and M Gass, J. Nucl. Mater. 465 (2015), p. 390.

[3] I MacLaren et al, Microscopy (2017) doi:10.1093/jmicro/dfx036.

[4] A Bashir et al, J. Appl. Phys., 123 (2018), p. 035703.

[5] CC Ahn and OL Krivanek in "EELS Atlas" (Arizona State University).

[6] KM acknowledges the funding from Helmholtz Association through a postdoctoral fellowship

a)

)

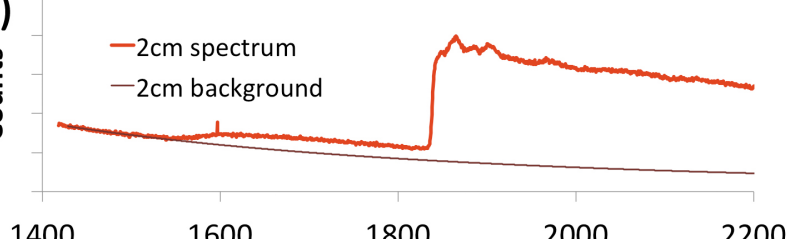

b)

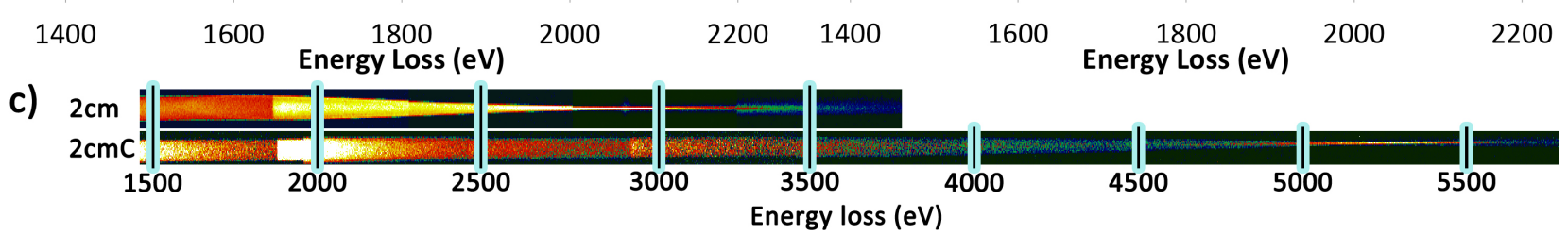

Figure 1. $80 \mathrm{kV}$ EELS characterization: a) a silicon K-edge recorded using the standard $2 \mathrm{~cm}$ camera length; b) the same spectrum recorded using the optimized $2 \mathrm{cmC}$ camera length; c) EELS stripe images from $\mathrm{Si}$ showing that at $2 \mathrm{~cm}$ camera length, a crossover is reached around $3500 \mathrm{eV}$, whereas this happens at about $5000-5500 \mathrm{eV}$ for the optimized $2 \mathrm{cmC}$ camera length.

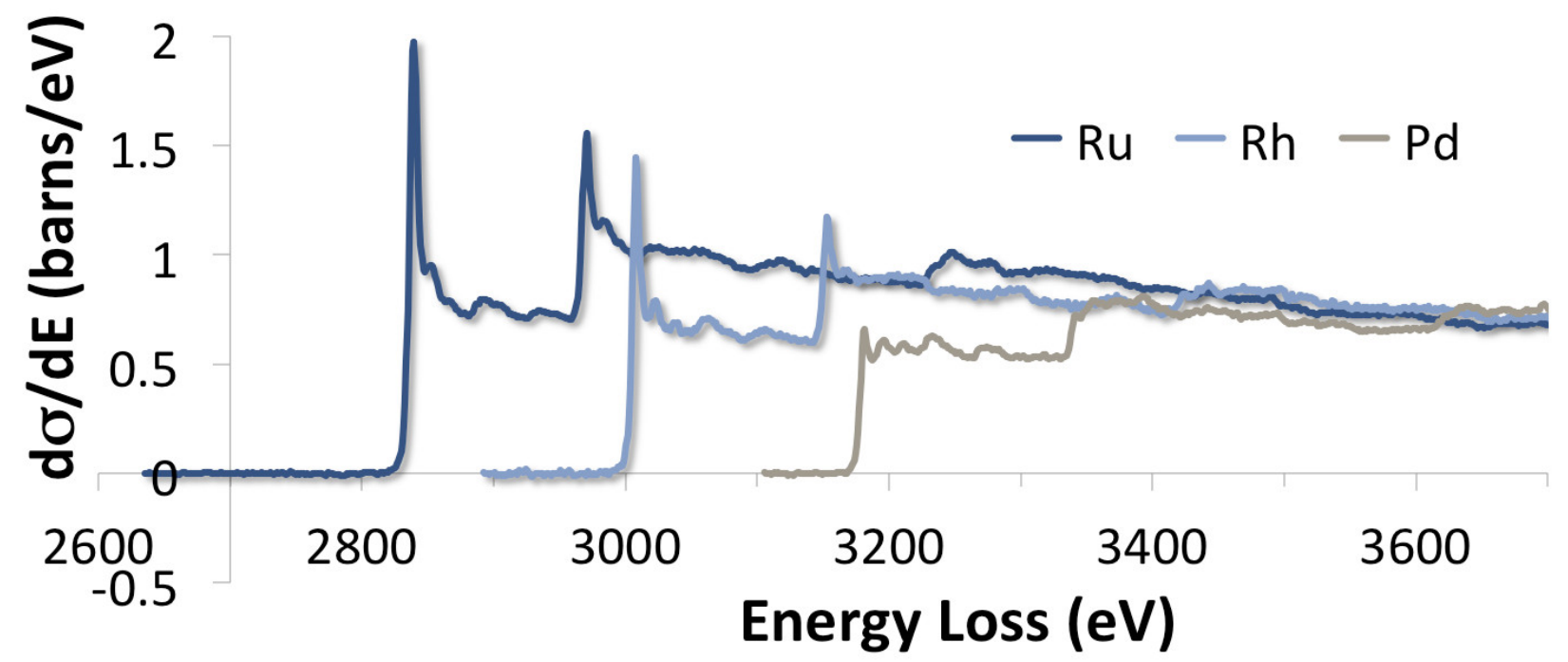

Figure 2. Absolute differential cross sections for three platinum group metals: $\mathrm{Ru}, \mathrm{Rh}$ and $\mathrm{Pd}$. 